\title{
X-ray cross-correlation analysis of the low-mass X-ray binary 4U 1636-53
}

\author{
Ya-Juan Lei \\ Key Laboratory of Optical Astronomy, National Astronomical Observatories, Chinese \\ Academy of Sciences, Beijing 100012, China \\ email: leiyj cwmy@163.com
}

\begin{abstract}
We analyze the cross-correlation function of the soft and hard X-rays of the atoll source $4 \mathrm{U}$ 1636-53 with $R X T E$ data. The results show that the cross-correlations evolve along the different branches of the color-color diagram. At the lower left banana states, we have both positive and ambiguous correlations, and positive correlations are dominant for the lower banana and the upper banana states. The anti-correlation is detected at the top of the upper banana states. The cross-correlations of two atoll sources $4 \mathrm{U} 1735-44$ and $4 \mathrm{U} 1608-52$ have been studied in previous work, and the anti-correlations are detected at the lower left banana or the top of the upper banana states. Our results show that, in the $4 \mathrm{U}$ 1636-53, the distribution of the cross-correlations in the color-color diagram is similar to those of $4 \mathrm{U} 1735-44$ and $4 \mathrm{U} 1608-52$, and confirm further that the distribution of cross-correlations in color-color diagram could be correlated with the luminosity of the source.
\end{abstract}

Keywords. X-rays: binary, accretion

\section{Introduction}

Low-mass X-ray binaries (LMXBs) with weakly magnetized neutron stars (NSs) can be classified into two subclasses, $\mathrm{Z}$ sources with high luminosity and atoll sources with low luminosity, according to their X-ray spectral and timing properties (Hasinger \& van der Klis 1989). In an X-ray color-color diagram (CCD), the Z source traces out a Z track within the shorter timescales (hours to days), whereas the typical atoll source shows a C-shaped track within days to weeks or so. It is suggested that most timing and spectral characteristics on the position along the $\mathrm{Z}$ or atoll track are related to the mass accretion rate (van der Klis 2000). With the mass accretion rate increasing, the atoll sources evolve from the island state (IS) to the banana branch, which is usually subdivided into lower left banana (LLB), lower banana (LB), and upper banana (UB) states.

Both the spectral and timing analyses are usually used to explore physical changes in the source emission. The cross-correlation function between different X-ray energy bands is also a useful means for studying the accretion disk. The anti-correlated lags of a few hundred seconds are found in some black hole X-ray binaries, $\mathrm{Z}$ and atoll sources. It is suggested that the large timescale of lags could correspond to the viscous timescales of the inner accretion disk (Sriram et al. 2012). In earlier work, we firstly detect the anticorrelations in two atoll sources $4 \mathrm{U} 1735-44$ and $4 \mathrm{U} 1608-52$, which occur in the LLB or the top of the UB. The cross-correlated results suggest that the observed anti-correlation may be relevant to the transition between the hard and soft states (Lei et al. 2013, 2014). It is obvious that more observations are needed to consolidate the inference. In the work, we study the cross-correlations of $4 \mathrm{U} 1636-53$ with $R X T E$ data. $4 \mathrm{U} 1636-53$ is one of the most interesting LMXBs of the atoll source, and it contains a companion star mass $\sim 0.4 M_{\odot}$ with an orbital period of $3.8 \mathrm{hr}$ (van Paradijs et al. 1990). We describe the observations in section 2 , show the results and discussion in section 3 . 



Figure 1. Light curves with bin size of $16 \mathrm{~s}$ and cross-correlation of the segment with anti-correlation detected. Symbol " a " corresponds to the individual segment for which anti-correlation is detected.


Figure 2. CCD and HID of $4 \mathrm{U}$ 1636-53 of the observation P10088. The cross stands for the positive-correlation, the triangle corresponds to the ambiguous and the circle indicates the anti-correlation. Each point is on average of $512 \mathrm{~s}$.

\section{Observations}

The observations of $4 \mathrm{U}$ 1636-53 analyzed in this paper are from the Proportional Counter Array (PCA) on board the RXTE satellite during April 1996 to February 1997 (P10088). PCA consists of 5 non-imaging, co-aligned Xe multiwire proportional counter units (PCUs). In this work, only PCU2 data are adopted. The light curves are extracted using standard2 data mode, with bin size of $16 \mathrm{~s}$. The XRONOS tool "crosscor" is used for estimating the cross-correlation between the soft X-rays (2.0-3.5 keV) and the hard X-rays (12-30 keV) for the observations whose the exposure time lasting longer than 2000 s. The data of type I X-ray bursts are excluded. For CCD analysis, the soft and the hard colors are defined as the count-rate ratios 3.5-6.0 keV/2.0-3.5 keV and 9.7-16 keV/6.0-9.7 $\mathrm{keV}$, respectively. In the hardness intensity diagrams (HID), the intensity in the energy band $2.0-16 \mathrm{keV}$ is calculated and is normalized to the Crab count rate.

In the same way as the previous work, the cross-correlation results are divided into three groups: positive, ambiguous and anti-correlated classes (Lei et al. 2008). An anticorrelation between the soft and hard X-rays refers to a negative cross-correlation coefficient, and vice versa for a positive correlation. Fig. 1 shows the background subtracted light curves, the anti-correlation between soft and hard X-rays for the observation with anti-correlation detected. The distribution of cross-correlations in the CCD of the analyzed observations is shown in Fig. 2. 


\section{Results and Discussion}

We have studied the cross-correlations between the soft and hard X-rays of 4U 1636-53 of the observation P10088 of RXTE and find that it is evolutional along the different branches of the CCD. Fig. 2 shows the distribution of cross-correlations in the CCD. One sees from Fig. 2 that, both positive and ambiguous correlations are detected at the LLB in similar proportion, but the positive correlations dominant for the LB and the UB. The anti-correlation of the soft and hard X-rays is detected at the top of the UB (ObsID 10088-01-06-08), where the time lag is about two hundred seconds (Fig. 1). Anticorrelated X-ray time lags of a few hundred seconds seen in some LMXBs suggest that the accretion disk could be truncated (Sriram et al. 2012). Therefore at the top of the $\mathrm{UB}$ of $4 \mathrm{U} 1636-53$ where the accretion rate is most highest, the accretion disk could be truncated in a radius very close to the NS.

For LMXBs, it is suggested that the cold photons come from the accretion disk, and the high energy photons from the hot corona. In the LLB, the lower accretion rate leads to the accretion disk boundary be away from the hot corona, and which creates the ambiguous and weakly positive correlations of the soft and hard X-rays. In the LB and the $\mathrm{UB}$, the accretion rate increases, and the accretion disk moves inward and is close to the hot corona, so the soft seed photons increase and can be Comptonized effectively. The increasing of both the soft and hard X-rays results in the positive correlation. However, when the luminosity is beyond a critical luminosity at the top of the UB, the Comptonizing cloud shrinks. The soft seed photons increase and cool the Compton cloud, which results in the decreasing of the hard photons, and hence the anti-correlation.

The distribution of cross-correlation in the CCD of $4 \mathrm{U} 1636-53$ is similar to those of atoll sources $4 \mathrm{U} 1735-44$ and $4 \mathrm{U}$ 1608-52, where anti-correlations are detected at the LLB or the top of the UB. The spectral analyses of $4 \mathrm{U} 1735-44$ and $4 \mathrm{U} 1608-52$ suggest that both the LLB and the top of the UB could be corresponding to the transition between the hard and soft states. The cross-correlated results of $4 \mathrm{U} 1636-53$ are also consistent with those of black hole X-ray binaries whose anti-correlations are also detected in the transition state.

The studies of $4 \mathrm{U} 1735-44$ and $4 \mathrm{U} 1608-52$ suggest that the position of the anticorrelation in the CCD can be related to the luminosity of the source, i.e., the anticorrelation is located at the top of the UB when the luminosity of a atoll source is lower, and occurs in the LLB when the luminosity is higher (Lei et al. 2013, 2014). For the observation P10088 of $4 \mathrm{U}$ 1636-53, the luminosity is lower and the anti-correlation is detected at the top of the UB, which is consistent with those of $4 \mathrm{U} 1735-44$ and $4 \mathrm{U}$ 1608-52. In addition, the study of XTE J1701-462 also implies that the position of anticorrelations in the CCD might depend on the luminosity of the source (Wang et al. 2014). Further work is needed in the future for exploring the relation.

\section{References}

Hasinger, G. \& van der Klis, M. 1989, $A \mathscr{\mho} A, 225,79$

Lei, Y. J., Qu, J. L., Song, L. M., et al. 2008, ApJ, 677, 461

Lei, Y. -J., Zhang, H. -T., Zhang, C. -M., et al. 2013, AJ, 146, 60

Lei, Y. -J., Zhang, S., Qu, J. -L., et al. 2014, AJ, 147, 67

Sriram, K., Choi, C. S., \& Rao, A. R. 2012, ApJS, 200, 16

van der Klis, M. 2000, ARAA, 38, 717

van Paradijs, J., van der Klis, M., van Amerongen, S., et al. 1990, A\&A, 234, 181

Wang, Y. N., Lei, Y. J., Ding, G. Q., et al. 2014, MNRAS, 440, 3726 